\title{
ENFOQUE COGNITIVO Y PSICOLINGÜÍSTICO DE LA LECTURA: DISEÑO Y VALIDACIÓN DE UNA PRUEBA DE HABILIDADES PRELECTORAS (THP) EN NIÑOS Y NIÑAS DE LA PROVINCIA CONSTITUCIONAL DEL CALLAO, PERÚ
}

\author{
COGNITIVE APPROACH AND PSYCHOLINGUISTICS OF READING: \\ DESIGN AND VALIDATION OF A TEST OF READING SKILLS IN THE CHILDREN OF \\ CALLAO, PERÚ \\ Esther Velarde C. ${ }^{1}$; Ricardo Canales G. ${ }^{2}$; Magali Meléndez J. ${ }^{3}$; Susana Lingán H. ${ }^{4}$ \\ Universidad Nacional Mayor de San Marcos, Perú \\ (RECIBIDO EL 20/01/2010, ACEPTADO EL 22/06/2010)
}

\begin{abstract}
RESUMEN
El presente estudio partió de la tesis psicolingüística y cognitiva que considera que para el aprendizaje de la lectura es indispensable haber alcanzado un nivel de desarrollo en la conciencia fonológica, el conocimiento del lenguaje escrito, la memoria verbal y los niveles semánticos y sintácticos del lenguaje oral. En tal sentido se planteó el estudio de dichas variables como predictoras del aprendizaje lector en niños ingresantes al primer grado de primaria en la región del Callao. Para cumplir dicho propósito se diseñó y validó el Test de habilidades prelectoras (THP) compuesto por cuatro subtest; el primero que evalúa la conciencia fonológica, el segundo que evalúa el conocimiento del nombre y el sonido de las letras, el tercero que evalúa memoria verbal y, finalmente, el cuarto que evalúa los componentes sintácticos y semánticos del lenguaje oral.
\end{abstract}

Se estimó la fiabilidad, mediante el alfa de Cronbach y el coeficiente de Spearman-Brown. Se determinó la validez de construcción mediante el análisis factorial. Finalmente se halló la validez predictiva correlacionándola con la Batería de evaluación de los procesos lectoresrevisada (Cuetos, 2007). Se ofrece al medio académico y profesional un instrumento que permitirá predecir el nivel de aprendizaje de la lectura para intervenir en aquellas áreas.

Palabras clave: Procesos psicolinguísticos; conciencia fonológica; test de habilidades prelectoras.

\begin{abstract}
This study was based on the psycholinguistics and cognitive theory believes that learning to read, candidates must have achieved a level of development in phonological awareness, knowledge of written language, verbal memory and semantic and syntactic levels of language oral. In this article we raised the consideration of these variables as predictors of learning reader in first grade children entering primary schools in the region of Callao. To fulfill that
\end{abstract}

1 Docente asociada e investigadora de la Facultad de Educación de la UNMSM.

E-mail: emvelarde@hotmail.com. 
purpose, we designed and validated TEST PRE-READING SKILLS (THP), consisting of four subtests, the first to evaluate phonological awareness, the second, which assesses knowledge of the name and sound of the letters, the third which assesses verbal memory, and finally, the fourth which assesses the syntactic and semantic components of oral language.

Reliability was estimated by Cronbach's alpha and Spearman-Brown coefficient. Was determined construct validity using factor analysis. Finally, predictive validity was found correlated with the Assessment Battery-Revised reading process (Cuetos F. 2007). It offers the professional academic and an instrument to predict the level of learning to read to intervene in areas deficient.

Keywords: Psycholinguistic processes; phonological awareness; test of prereading skills.

\section{INTRODUCCIÓN}

En el Perú tenemos un grave problema en relación al aprendizaje de la lectura. A los resultados mostrados por el Laboratorio latinoamericano de evaluación de la calidad educativa (1997 y 2006), en donde nuestros niños de 3 er $^{\text {e }} 4 .{ }^{\text {to }}$ grado de primaria siguen ocupando los últimos lugares en la región, y el estudio de la Prueba PISA (2003), donde un $54 \%$ de nuestros estudiantes de secundaria - con promedio de edad de 15 añosalcanza un nivel 0 en competencias lectoras; se añade el último estudio realizado por el propio Ministerio de Educación (2007), en donde se indica que en la evaluación sobre lectura y comprensión de textos, en una muestra nacional de alumnos de segundo grado de primaria, solo el $15.9 \%$ podía lograr ejecutar tareas esperadas para su grado, el $54 \%$ podía desempeñar solo tareas de menor dificultad y el restante $29.8 \%$ no podía lograr ni siquiera eso.

Años atrás, en uno de sus últimos estudios, González, R. (2006), habiendo examinado los niveles de comprensión lectora a 103 alumnos con la técnica de cloze, encontró que los promedios hallados se encontraban por debajo del nivel crítico, indicando analfabetismo funcional. Esto explicaría el bajo rendimiento académico obtenido por los estudiantes debido a que la lectura constituye la herramienta fundamental para el aprendizaje escolar.

De otro lado, en los últimos años, el Banco Mundial (2006) ha señalado que, en cuanto a lectura, los niveles de velocidad lectora de nuestros niños son muy preocupantes. Siendo el estándar internacional para países en vías de desarrollo el siguiente: primer grado, 30 palabras por minuto; segundo grado, 60 palabras; tercer grado, 90 palabras; cuarto grado 110 palabras; se ha observado que en promedio nuestros niños en primer grado de primaria están leyendo tan solo 9 palabras por minuto, y los de segundo grado, 29 palabras. Tal es pues la gravedad de este problema educativo en nuestro país.

En los últimos años se viene tomando conciencia progresivamente de la gravedad del problema. Quizá no hemos llegado aún al nivel de preocupación que el gobierno y las autoridades de países vecinos (Uruguay y Chile) expresan al respecto, al punto de abordar dicha problemática con políticas de estado, pero por lo menos en el campo de la investigación estamos avanzando. El problema central radica en encontrar las variables que están directamente relacionadas con el éxito en el aprendizaje de la lectura. Al respecto, Rosales (1984) trató de establecer si existía algún grado de relación entre el contexto social 
(medido como número total de habitantes de un núcleo urbano específico) y el rendimiento en comprensión lectora en una muestra de sujetos de 9 a 13 años pertenecientes a núcleos urbanos cerca de Lima. Los resultados hallados indican que el tamaño de la población, así como la mayor interacción social, intelectual y cultural influían directamente en la comprensión lectora.

En los últimos años, Velarde y Canales (Bravo, L., 2009) vienen profundizando el estudio de la lectura bajo un enfoque cognitivo y psicolingüístico. Velarde (2001) encontró que en niños que cursaban el tercer grado de primaria en el Cercado del Callao se daba una relación entre los niveles de conciencia fonológica y la lectura (decodificación y comprensión). Aplicando la prueba de conciencia fonémica (PCF) de Jiménez y la prueba de análisis fonémico (PAF) de Ortiz, el TEDE de Condemarín y Blomquist, y la prueba de comprensión lectora (CLP) de Condemarín, halló que en el nivel socioeconómico bajo existía una correlación altamente significativa entre el nivel de conciencia fonémica y la decodificación lectora, y una correlación muy baja entre la conciencia fonémica y la comprensión lectora. En el nivel socioeconómico medio se encontró igualmente correlación altamente significativa entre la conciencia fonémica e intrasilábica (conciencia fonológica) y la decodificación, con la diferencia que también se halló relación altamente significativa entre dichas variables fonológicas y la comprensión lectora.

Velarde y Canales (2008) hallaron relación entre habilidades psicolingüísticas de base y dominio de la lectura en niños de segundo grado de primaria de las zonas pobres del Callao ("Mi Perú"-Ventanilla). Encontraron relación entre el nivel lingüístico y metalingüístico del lenguaje oral y el dominio de la lectura. Además se halló que el rendimiento de los niños (as) de las instituciones educativas estatales más pobres era significativamente inferior al rendimiento de los niños (as) igualmente pobre pero que estudiaban en una institución educativa parroquial ("Fe y Alegría”). La diferencia en el rendimiento estaría relacionada con el clima institucional, la calidad de la enseñanza y la gestión educativa.

Por otro lado, también, se encuentran las investigaciones referidas a programas de intervención para el mejoramiento de la lectura. Así, Tapia (1996) realizó una investigación de evaluación formativa sobre las bondades de un Programa de desarrollo de la comprensión lectora en niños deficientes lectores de cuarto y quinto grado de nivel socioeconómico bajo. Para evaluar el grado de comprensión lectora se utilizó la Prueba de complejidad lingüística progresiva de Condemarín, Milicic y Alliende (Chile, 1990). Los resultados demostraron que los niños deficientes lectores no solo superaron el déficit encontrado al inicio de la aplicación del programa, sino que incluso superaron significativamente al grupo de buenos lectores (esto se halló en el subgrupo de niños deficientes lectores que pertenecían al quinto grado).

Por otro lado, respecto a la relación entre habilidades metacognitivas y lectura, Pinzás (1997) cita los trabajos de Haller et al. (1988) en donde se examinan los efectos de la enseñanza de habilidades metacognitivas sobre la comprensión lectora. En una muestra total de 1553 niños, en donde se usó grupo control y se aplicaron los procedimientos estadísticos de rigor, se encontró que el entrenamiento en este tipo de habilidades era más efectivo. Demostró que la conciencia metacognitiva y la ejecución de acciones de monitoreo se vio mejorada con el uso de la técnica de provocar las disonancias cognitivas. 
Canales (2007) realiza otra investigación en el campo de la comprensión lectora; partiendo del modelo teórico de la psicología cognitiva y la psicolingüística, en particular de la teoría de Van Dijk y Kintsch $(1983,1993,1994)$, el autor explora la problemática de la lectura comprensiva en una muestra de niños y adolescentes con problemas de aprendizaje de la provincia del Callao, tratando de conocer hasta qué punto se puede intervenir en la mejora de sus deficiencias lectoras, para lo cual diseñó y aplicó un Programa experimental de tratamiento. Como hallazgo fundamental del estudio se encontraron diferencias significativas en los niveles de comprensión lectora alcanzados en el pretest y el postest por los alumnos del agrupamiento I (secundaria) expuestos al programa experimental, así como incremento importante en los alumnos del agrupamiento II (primaria).

Velarde (2008) aplicó un programa de habilidades metafonológicas en niños de tercer y cuarto grado de primaria del nivel socioeconómico bajo y demostró la efectividad del Programa sobre la conciencia fonológica, la decodificación lectora y la comprensión.

Velarde, Meléndez, Canales y Lingán (2009) aplicaron un programa cognitivo y psicolingüístico para el mejoramiento de la conciencia fonológica, memoria verbal, conocimiento de letras y el lenguaje oral en colegios del Callao y observaron progresos significativos en los niños de educación inicial en las habilidades prelectoras mencionadas.

\section{El enfoque cognitivo y psicolingüístico de la lectura}

Frente al tipo de dificultades serias que nuestros alumnos presentan en el aprendizaje de la lectura habría que empezar a preguntarnos acerca de las causas. En los últimos años, nuevos enfoques e investigaciones vienen enriqueciendo la comprensión de lo que ocurre en la mente del niño o la persona que sufre dificultades en la lectura. Bravo (2005), reafirmando la importancia de los procesos psicolingüísticos, señala que los procesos cognitivos que fallarían en aquellos serían los que permiten que la información visual se convierta en información verbal y sea luego transferida hacia los niveles superiores, que es donde finalmente adquiere significado. Para ese sentido se ha planteado un modelo cognitivo llamado: "arquitectura funcional" que explica el sistema de la lectura a través de tres procesos:

a) Procesos de bajo nivel: es el nivel de procesamiento que se encarga del recojo de la información gráfica a través de los movimientos oculares de fijación y saccádicos que permiten extraer la información de las letras (rasgos), transformarla en códigos y almacenarla brevemente (apenas unos milisegundos) en la memoria sensorial (memoria icónica). Luego, la información pasa a la memoria de corto plazo (MCP); aquí, la permanencia de la información es mucho mayor que en la memoria icónica. La MCP es capaz de almacenar 6 ó 7 estímulos visuales que almacena categorialmente como material lingüístico. Para que se pueda realizar el reconocimiento lingüístico, el lector consultará en su almacén de largo plazo, donde están codificadas todas las letras de su idioma.

b) Procesos de nivel medio: es el nivel de reconocimiento o de acceso al léxico. En este nivel se transforman las representaciones ortográficas en significados (siempre y 
cuando exista un conocimiento previo de esta palabra en el almacén léxico interno). Permite enfrentar la palabra a través de dos vías: una que conecta directamente los signos gráficos con sus significados (la estrategia visual) y otra que transforma los signos gráficos en sonidos (estrategia subléxica o fonológica).

c) Procesos de alto nivel: es el nivel encargado del procesamiento de textos a través de dos operaciones: el sintáctico y el semántico. El procesamiento sintáctico se encarga de poner en funcionamiento las claves gramaticales que nos informan de cómo se encuentran relacionadas las palabras y, además, reconocer la estructura de la oración. El nivel semántico es responsable de procesar el contenido conceptual y proposicional de las oraciones; además, relaciona las proposiciones para comprender la estructura global del significado del texto, extrae el mensaje del texto escrito y lo asimila a su estructura cognitiva que se encuentra en la memoria de largo plazo.

Estos sistemas se ponen en funcionamiento en la lectura. Pero nos preguntamos: ¿cuáles son las habilidades que deberían estimularse antes de la lectura?

Durante décadas se pensó que las funciones cognitivas relacionadas con el aprendizaje lector estaban relacionadas con la percepción visual y las habilidades psicomotoras (Jiménez, J.; Ortiz, M. 1998). Diversas pruebas de diagnóstico de la lectura se centraban en evaluar estas áreas pensando que estas variables predecían el éxito en el aprendizaje de la lectura. En las últimas décadas, múltiples investigaciones (Dominguez, A., 1999; Cuetos, V., 2008) realizadas en Europa, EE.UU. y Latinoamérica han demostrado que las habilidades que están directamente relacionadas con la lectura tienen que ver directamente con funciones lingüísticas y metalingüísticas (en especial, la conciencia fonológica, y el dominio sintáctico y semántico del lenguaje oral), y algunas funciones cognitivas también relacionadas con el lenguaje como la memoria verbal. A este enfoque teórico se le conoce como el Modelo cognitivo y psicolingüístico de la lectura. Además se han realizados estudios prelectores (Bravo, Villalón y Orellana, 2003) que también relacionan el éxito en la lectura con el reconocimiento previo de cinco letras como mínimo, con un poder predictor que alcanza hasta el tercer grado de primaria.

En este sentido y tomando en cuenta este aporte teórico e investigativo, se ha elaborado un Test de habilidades prelectoras: THP, que incluye el conjunto de estas variables demostradas como predictoras de la lectura tratando de superar a aquellos instrumentos que solo evalúan algunas de estas variables de manera individual. El objetivo central de esta prueba es detectar a los alumnos (as) que antes del aprendizaje de la lectura muestren dificultades, de tal manera que se pueda intervenir oportunamente en aquella habilidad que requiera refuerzo con el fin de garantizar el éxito en el aprendizaje de la lectura y formar una sociedad de lectores con sólidas herramientas cognitivas que les permitan enfrentar las demandas del siglo XXI. En tal sentido, nuestro instrumento de evaluación (THP) está compuesto por 4 subtests:

\section{Subtest de conciencia fonológica}

La conciencia fonológica es una habilidad metalingüística relacionada con la capacidad de manipular y reflexionar sobre los aspectos sonoros del lenguaje hablado. Cuando el niño (a) es capaz de tomar conciencia sobre un aspecto específico del lenguaje 
hablado estaremos hablando de la conciencia fonológica. La conciencia fonológica no es una entidad homogénea (Treiman, 1990), pues existen diferentes niveles de conciencia fonológica, dependiendo del tipo de unidad lingüística sobre la cual el niño aplica la reflexión y la manipulación de los sonidos del habla. Estos niveles son: rimas, sílabas, intrasílabas y fonemas. Cuando el sujeto es capaz de tomar conciencia sobre una estructura de la palabra que es mayor que la sílaba, está demostrando un nivel de dominio de la conciencia de rimas. Cuando es capaz de reflexionar sobre la estructura silábica de la palabra, estará realizando una conciencia silábica. Cuando opera sobre las unidades intrasilábicas (el onset y la rima), estará aplicando un nivel de conciencia intrasilábica. Y cuando reflexiona directamente sobre el fonema, estará desarrollando un nivel de conciencia fonémica.

La habilidad fonológica que tiene mayor correlación con la lectura es la conciencia fonémica; sin embargo, es la más compleja por el hecho de que los fonemas son unidades lingüísticas que aparecen coarticulados en las palabras y es más difícil su segmentación (Cuetos, 2008). No obstante, evaluaremos también las habilidades que anteceden al dominio fonémico (a excepción de la conciencia intrasilábica) que nos permitirán evidenciar el grado de desarrollo global de la conciencia fonológica, para evitar convertir la prueba en un instrumento tedioso y complejo.

El subtest de conciencia fonológica intenta evaluar la capacidad de reconocimiento que tiene el niño (a) sobre los sonidos en rimas, sílabas y fonemas, para culminar con tareas más complejas como la de segmentar y sintetizar fonemas.

\section{Subtest de conocimiento de letras}

Se ha considerado que otra habilidad predictiva del aprendizaje lector es el conocimiento de las letras antes de su enseñanza formal. En un estudio realizado por Bravo, Villalón y Orellana (2000) se demostró que los niños que al iniciar primer grado de educación primaria ya habían adquirido conocimiento de algunas letras (ya sea de su nombre o sea de su pronunciación) fueron mejores lectores en los primeros grados de primaria.

Por su parte, Foulin (2005) publicó un estudio sobre el efecto predictivo del conocimiento de las letras y el éxito en lectura. Sostuvo que el conocimiento de las letras en educación inicial es el mejor predictor individual de la lectura en primer año junto con la segmentación del primer fonema. El mejor resultado lo obtuvo combinando el conocimiento del nombre de las letras y de su sonido. Considera que el conocimiento de las letras permite establecer un puente desde la etapa logográfica de la lectura hasta la etapa alfabética donde se hace uso de la estrategia fonológica para la decodificación inicial. Este puente se puede establecer solo si los niños(as) han desarrollado un progresivo dominio de la conciencia fonológica. Por su parte, Compton (2000) encontró que los niños que en educación inicial conocen el nombre de las letras e identifican el fonema inicial de las palabras tienen mayor éxito en la lectura en el primer año. Muter y Snowling (1998), encontraron que las pruebas fonológicas (suprimir el primer fonema) junto con el conocimiento de las letras en educación inicial predijeron la lectura a los 9 años. Sin embargo, encontró que antes de esa edad no fueron predictivas. 
En resumen, estas investigaciones demuestran que el número de letras que conoce el niño (a) presenta un alto grado de predictividad sobre el aprendizaje de la lectura. Este grado de predictividad aumenta si el conocimiento de la letra va asociada con la identificación fonológica. Es decir que no basta con identificar las letras como figuras gráficas sino que, además, es necesario asociarlas con los sonidos de ellas (Guardia, 2002). En ese sentido, en esta prueba evaluaremos el reconocimiento tanto del nombre, como del sonido de la letra.

\section{Subtest de memoria verbal}

La memoria verbal es una habilidad cognitiva que atraviesa transversalmente todo el proceso de aprendizaje de la lectura. La memoria verbal de corto plazo se encarga de almacenar la información verbal que, como sabemos, dura aproximadamente 30 segundos y está basada en la ley descubierta por Miller conocida como el "número mágico 7+-2" que fundamenta la capacidad de retención de la información que oscila entre $5(7-2)$ y $9(7+2)$ unidades de información factibles de ser almacenadas por el sujeto, más aun si emplea estrategias mnemotécnicas. En el caso de la lectura es fundamental que esta memoria se encuentre en muy buenas condiciones, puesto que todas las tareas ejecutadas requieren la retención de diversos estímulos verbales como grafemas, fonemas, palabras, oraciones y texto. En esta prueba se han seleccionado una serie de oraciones que contienen desde 2 hasta 13 palabras que el sujeto, luego de escucharlas, debe almacenar y verbalizar de manera correcta sin omitir, adicionar, sustituir o transponer ninguno de los elementos que conforman la oración.

\section{El dominio semántico y sintáctico del lenguaje oral}

El lenguaje oral materno es un excelente predictor de las habilidades de la lectura relacionadas más directamente con la comprensión lectora (González, R., 2006). En el lenguaje oral intervienen varios tipos de procesamiento: uno de ellos es el procesamiento sintáctico y, el otro, es el procesamiento semántico.

Las habilidades sintácticas están relacionadas con el dominio estructural del lenguaje que es un tipo de procesamiento psicolingüístico que permite entender este a través de la relación existente entre las palabras. Las palabras no existen aisladas unas de otras, sino que guardan relación entre sí. De lo que se trata es de captar dicha relación, identificando la importancia funcional y la relación gramatical de cada palabra. Ello es posible gracias al papel de los analizadores sintácticos. En esta prueba evaluaremos el nivel sintáctico a través de tareas en las que el sujeto deberá hacer uso de palabras que juegan un rol sintáctico en la oración.

El procesamiento semántico es un tipo de procesamiento psicolingüístico que permite acceder al significado de las palabras y frases que se escuchan, captando el sentido global del mensaje o discurso. Actúa el subproceso de extracción del significado que consiste en construir la representación mental de la información oída, asignando los papeles específicos de actuación a los personajes que intervienen y el de integración del significado a la estructura de conocimientos que posee el oyente. Para que esto pueda ser posible, es necesario establecer un vínculo entre la nueva estructura (información nueva) y los conocimientos que ya posee el oyente (información dada). 
Para evaluar la capacidad de extraer el significado utilizaremos dibujos y oraciones donde el sujeto tendrá que discernir cuál de las ilustraciones corresponde a la expresión lingüística emitida oralmente por el examinador, para lo cual tendrá que asignar los papeles de agente de la acción (¿quién?), objeto de la acción (¿hizo qué?), lugar donde ocurre la acción (¿dónde?), momento en que ocurre la acción (¿cuándo?), etc.; también le pediremos al niño(a) que defina conceptos, obteniendo mayor puntaje aquel que exprese una respuesta categorial señalando características y que responda preguntas de comprensión oral realizando inferencias. El nivel sintáctico es evaluado a través de tareas donde el niño(a) deberá expresar una oración de forma correcta usando los pronombres y palabras funcionales solicitados.

\section{MÉTODO}

\section{Tipo de investigación}

La investigación es de tipo tecnológica.

\section{Participantes}

La muestra a la cual se aplicó el instrumento final estuvo conformada por 200 niños, provenientes de 8 escuelas públicas y privadas de la región Callao. El grado educativo de los sujetos constituyó el principal criterio de selección de la muestra, de tal forma que esta estuvo integrada por niños y niñas que estaban iniciando el primer grado de educación primaria, siendo la edad media de 5 años, 8 meses dentro de un rango que va desde los 5 años como mínimo hasta los 7 años, 6 meses como máximo. Asimismo se consideró dentro de la muestra cuatro niveles socioeconómicos tomando como criterio el tipo de escuela - pública o privada - y el lugar de procedencia de los alumnos. Las escuelas fueron seleccionadas de forma aleatoria dentro del conjunto de instituciones educativas que cumpliesen con los criterios mencionados. Así también se trató de obtener aproximadamente una muestra equilibrada entre alumnos de sexo femenino y masculino.

\section{Instrumento}

El Test de habilidades prelectoras (THP) está conformado por 117 ítemes agrupados en 15 tareas, que se reúnen a su vez en 5 dimensiones. Cada una de ellas está teóricamente definida de la siguiente manera: conciencia fonológica, conocimiento de letras, memoria verbal, procesos sintácticos del lenguaje oral y procesos semánticos del lenguaje oral.

\section{Procedimiento}

Para el diseño y la elaboración del Test de habilidades prelectoras (THP) se realizó un abordaje teórico y conceptual desde la perspectiva psicolingüística del aprendizaje de la lectura. El THP estuvo conformado en un primer momento por 156 reactivos con distintas tareas agrupadas en las cinco dimensiones ya explicadas. 
En un primer análisis de los reactivos se seleccionó a una muestra piloto constituida por los 10 niños más hábiles que iniciaban el primer año de educación primaria, procedentes de una institución educativa particular con un exigente nivel educativo. Este procedimiento se realizó con el objetivo de depurar aquellos reactivos de mayor dificultad y no apropiados para la muestra objetivo, además que dicha evaluación permitió realizar un examen cualitativo de la prueba, mejorando las instrucciones y los reactivos que pudiesen ser confusos.

Luego de la evaluación piloto y tras el análisis estadístico de los resultados, se eliminó el conjunto de ítemes correspondientes a la tarea de análisis fonémico, puesto que ningún sujeto evaluado logró realizar con éxito dicha tarea a pesar de que se trataba de un grupo altamente calificado. De esta manera, el THP se redujo a 148 reactivos.

Posteriormente se procedió a la evaluación individual de los 200 sujetos integrantes de la muestra con la nueva versión del THP, los resultados arrojados fueron analizados con el objetivo de obtener los fundamentos estadísticos para la confiabilidad y validez de la prueba, a partir de estos análisis se realizaron los ajustes y afinamientos necesarios para obtener finalmente la última versión del THP con 117 reactivos.

Por último, con el objetivo de obtener la validez predictiva del instrumento, se procedió a evaluar a los 200 estudiantes de la muestra con la Batería de evaluación de los procesos lectores, ed. revisada (PROLEC-R) de F. Cuetos, B. Rodríguez, E. Ruano y D. Arribas (2007) en el último trimestre del año escolar. Los datos obtenidos fueron analizados con el paquete estadístico SPSS versión 17.

\section{RESULTADOS}

\section{Confiabilidad}

Para el análisis de fiabilidad se ha considerado la perspectiva de la consistencia interna, para lo cual se calcularon los valores de los coeficientes de alfa de Cronbach (Tabla 1), así como la correlación de cada ítem componente con el total de la tarea, obteniendo el coeficiente producto-momento de Pearson correspondiente. Los reactivos con correlación menor a 0.40 fueron eliminados, de esta manera el número de ítemes del THP se redujo a 117.

La consistencia interna del THP alcanzó un valor alpha de 0.964 para la prueba total, y la retirada de ninguno de sus ítemes permitió mejorar dicho valor. Así también, los valores de la correlación de cada uno de los ítemes con el puntaje total se situaron por encima del valor de 0.40. Al realizar el análisis de confiabilidad de las sub-escalas se encontraron valores de alfa aceptables que oscilan entre 0.567 y 0.935 . Cabe señalar que en las tareas en donde se han obtenido valores de alfa por debajo de 0.65 , estos responden a características propias de las pruebas tipo screening que poseen un número reducido de reactivos y con una escasa dificultad, lo que conlleva a una baja covariación entre los elementos. Por lo tanto, considerando lo anterior, se puede afirmar que los valores alfa son satisfactorios.

Tabla N. ${ }^{0}$ 1. Coeficientes de consistencia interna. 


\begin{tabular}{llll}
\hline \multicolumn{1}{c}{ Subtest } & \multicolumn{1}{c}{ Tareas } & Alfa & N. $^{\text {o }}$ de reactivos \\
\hline Conciencia fonológica & rimas & 0.791 & 6 \\
& sílaba inicial & 0.740 & 5 \\
& sílaba final & 0.736 & 4 \\
& sílaba medial & 0.731 & 5 \\
& fonema inicial & 0.721 & 5 \\
& fonema final & 0.682 & 5 \\
& fonema medial & 0.695 & 5 \\
Conocimiento de letras & níntesis fonémico & 0.924 & 8 \\
& nombre de letras & 0.913 & 15 \\
Memoria verbal & ronido de letras & 0.935 & 15 \\
Procesos sintácticos & retención y repetición y reconocimiento & 0.572 & 7 \\
Procesos semánticos & procesos sintácticos & 0.628 & 10 \\
& nivel conceptual & 0.557 & 8 \\
& comprensión oral & 0.646 & 10 \\
\hline \multirow{2}{*}{ total } & 0.964 & 117 \\
\hline & & & 9 \\
\hline
\end{tabular}

\section{Validez de constructo}

\section{Análisis factorial}

La medida de adecuación muestral de Kaiser-Mayer-Olkin (0.90) y la prueba de esfericidad de Bartlett con valores de 15240.239 ( $\mathrm{Sig} .=.000)$ indicaron que era posible el análisis factorial de la matriz de correlaciones; para ello se utilizó el método de componentes principales con rotación varimax. Se obtuvieron 15 factores que explican el $70.68 \%$ de la varianza total con pesos factoriales y comunalidades por encima del valor de 0.60. Asimismo se realizó el análisis factorial por subtest, encontrándose que cada dimensión es preferentemente unidimensional de acuerdo al criterio establecido por Carmines y Zeller (1979), el cual afirma que un conjunto de ítemes será unidimensional si el primer factor explica por lo menos el $40 \%$ de la varianza total.

Tabla N. ${ }^{0}$ 2. Estructura factorial del THP. 


\begin{tabular}{|c|c|c|c|}
\hline \multirow{2}{*}{ Factor } & \multicolumn{2}{|c|}{ Varianza total explicada } & \multirow{2}{*}{$\begin{array}{l}\text { Ne ítemes } \\
\text { demes }\end{array}$} \\
\hline & $\begin{array}{c}\% \\
\text { de la varianza }\end{array}$ & $\begin{array}{l}\% \text { acumulado } \\
\text { de la varianza }\end{array}$ & \\
\hline Factor 1: identificación del nombre y sonido de las consonantes. & 24.443 & 24.443 & 20 \\
\hline Factor 2: síntesis fonémica. & 4.198 & 28.641 & 8 \\
\hline Factor 3: identificación del fonema común al inicio, final y medio de una palabra. & 3.718 & 32.359 & 10 \\
\hline Factor 4: identificación de la sílaba común al inicio, final y medio de una palabra. & 3.059 & 35.418 & 9 \\
\hline Factor 5: identificación de sílaba o fonema diferente al inicio, final y medio de una palabra. & 2.507 & 37.925 & 10 \\
\hline Factor 6: identificación del nombre y sonido de las vocales. & 2.429 & 40.354 & 10 \\
\hline Factor 7: conocimiento de conceptos abstractos. & 2.063 & 42.41 & 3 \\
\hline Factor 8: conocimiento de conceptos concretos. & 2.021 & 44.438 & 7 \\
\hline Factor 9: comprensión inferencial. & 1.915 & 46.353 & 3 \\
\hline Factor 10: comprensión literal. & 1.871 & 48.224 & 6 \\
\hline Factor 11: repetición de oraciones de 5 o menos palabras. & 1.723 & 49.947 & 2 \\
\hline Factor 12: repetición de oraciones de más de 5 palabras. & 1.655 & 51.602 & 5 \\
\hline Factor 13: procesos sintácticos. & 1.550 & 53.152 & 8 \\
\hline Factor 14: identificación de rimas. & 1.522 & 54.674 & 6 \\
\hline Factor 15: retención y evocación de palabras. & 1.444 & 56.118 & 10 \\
\hline
\end{tabular}

Tal como se aprecia en el Tabla 2, la estructura factorial difiere de la estructura planteada en la construcción de la prueba; sin embargo, ambas distribuciones no son incompatibles y sus diferencias obedecen más a la importancia que el análisis factorial da a la distribución de las puntuaciones que a una discordancia con el modelo teórico subyacente. Por ende se puede decir que ambas distribuciones son funcionales en la realidad empírica. Esto último permite que se tome la estructura planteada en un primer momento con fines prácticos y para lograr un ordenamiento de los ítemes más comprensibles para el uso de la prueba.

\section{Validez de criterio externo}

Uno de los objetivos principales, en cuanto a la validez de la prueba, fue corroborar su poder predictivo. Para ello se utilizó como criterio la Batería de evaluación de los procesos lectores, ed. revisada (PROLEC-R) (F. Cuetos, B. Rodríguez, E. Ruano y D. Arribas, 2007), prueba confiable utilizada para la identificación de las dificultades en cada uno de los procesos de la lectura, incluidos en ellos: procesos perceptivos, léxicos, sintácticos y semánticos. Se aplicaron 6 subtests de dicha prueba: identificación de letras, lectura de palabras, lectura de pseudopalabras (los dos últimos subtest forman parte del factor de procesos léxicos), estructura gramatical (procesos sintácticos), comprensión de oraciones y comprensión de textos (procesos semánticos).

Tras la aplicación del PROLEC-R, en el último trimestre educativo, a los 200 sujetos integrantes de la muestra, los resultados arrojados muestran correlaciones significativas entre los subtest del THP y los subtest del PROLEC-R (Tabla N. ${ }^{\circ}$ 3). El análisis 
correlacional fue realizado a través del estadístico rho de Spearman, ya que al ser el PROLEC-R una prueba destinada a la detección de dificultades en cada uno de los procesos lectores, la media de la muestra - en la mayoría de los subtest - fue cercana a la máxima teórica alcanzable. Entre los resultados se destaca que en la mayoría de los casos las correlaciones son significativas al nivel 0.01. Cabe señalar que en el subtest de conciencia fonológica del THP, la tarea con los coeficientes más altos, y por lo tanto la que posee mayor asociación con el PROLEC-R, es la de síntesis fonémica. Asimismo, importantes correlaciones se encuentran entre las tareas referidas a la identificación de letras (identificación del nombre y del sonido) del THP y los subtests del PROLEC-R. Además existe una alta correlación entre la tarea de lenguaje oral del THP y los procesos semánticos de la lectura del PROLEC-R constituido por las tareas de comprensión de oraciones y comprensión de textos.

Tabla N. ${ }^{\circ}$ 3. Correlaciones del THP y el PROLEC-R.

\begin{tabular}{|c|c|c|c|c|c|c|}
\hline \multirow{2}{*}{$\begin{array}{c}\text { Subtest } \\
\text { THP }\end{array}$} & & \multicolumn{5}{|c|}{ PROLEC-R } \\
\hline & & $\begin{array}{l}\text { Id. de } \\
\text { letras }\end{array}$ & $\begin{array}{l}\text { Proc. } \\
\text { léxicos }\end{array}$ & $\begin{array}{l}\text { Estructura } \\
\text { gramatical }\end{array}$ & $\begin{array}{c}\text { Proc. } \\
\text { semánticos }\end{array}$ & Total \\
\hline & Alfa & 0,927 & 0,991 & 0,910 & 0,960 & 0,901 \\
\hline \multirow{8}{*}{ Conciencia fonológica } & Rimas & $0.274 * *$ & 0.139 & $0.159 *$ & $0.171^{*}$ & $0.175^{*}$ \\
\hline & Sílaba inicial & $0.400 * *$ & $0.401 * *$ & $0.380 * *$ & $0.417 * *$ & $0.435 * *$ \\
\hline & Sílaba final & $0.485^{* *}$ & $0.434 * *$ & $0.411 * *$ & $0.495^{* *}$ & $0.489 * *$ \\
\hline & Sílaba medial & $0.432 * *$ & $0.347 * *$ & $0.366^{* *}$ & $0.389 * *$ & $0.404 * *$ \\
\hline & Fonema inicial & $0.488 * *$ & $0.389 * *$ & $0.466 * *$ & $0,479 * *$ & $0.467 * *$ \\
\hline & Fonema final & $0.381 * *$ & $0.325 * *$ & $0.349 * *$ & $0.385 * *$ & $0.377 * *$ \\
\hline & Fonema medial & $0.497 * *$ & $0.434 * *$ & $0.393 * *$ & $0.499 * *$ & $0.491 * *$ \\
\hline & Síntesis fonémico & $0.533 * *$ & $0.532 * *$ & $0.471 * *$ & $0.576^{* *}$ & $0.564 * *$ \\
\hline \multirow{2}{*}{ Id. de letras } & Nombre de letras & $0.658^{* *}$ & $0.609 * *$ & $0.484 * *$ & $0.605^{* *}$ & $0.644 * *$ \\
\hline & Sonido de letras & $0.551 * *$ & $0.506^{* *}$ & $0.465 * *$ & $0.550 * *$ & $0.528 * *$ \\
\hline \multirow{2}{*}{ Memoria verbal } & Retención y repetición & $0.270^{* *}$ & $0.210^{* *}$ & $0.276^{* *}$ & $0.293 * *$ & $0.289 * *$ \\
\hline & Retención y reconocimiento & 0.115 & 0.073 & $0.162 *$ & $0.189^{*}$ & $0.176^{*}$ \\
\hline Procesos sintácticos & Procesos sintácticos & $0.368 * *$ & $0.369 * *$ & $0.371 * *$ & $0.410^{* *}$ & $0.416 * *$ \\
\hline \multirow{3}{*}{ Procesos semánticos } & Nivel conceptual & $0.349 * *$ & $0.235^{* *}$ & $0.274 * *$ & $0.250^{* *}$ & $0.290 * *$ \\
\hline & Comprensión oral & $0.370 * *$ & $0.335^{* *}$ & $0.462 * *$ & $0.498 * *$ & $0.439 * *$ \\
\hline & Total & $0.595 * *$ & $0.531 * *$ & $0.533 * *$ & $0.601 * *$ & $0.605^{* *}$ \\
\hline
\end{tabular}

* La correlación es significativa al nivel 0.05 .

** La correlación es significativa al nivel 0.01 . 
Como se ha podido observar, efectivamente existe una asociación bastante aceptable entre las puntuaciones alcanzadas por la muestra al inicio de año en el THP y al final del mismo en el PROLEC-R, lo que demostraría que el THP posee un buen nivel de predicción de la lectura.

\section{DISCUSIÓN}

El Test de habilidades prelectoras (THP) es un instrumento de orientación cognitiva y psicolingüística que nos permite evaluar las variables que preceden al aprendizaje de la lectura. Estas habilidades constituyen una manera distinta de abordar la problemática de la enseñanza de la lectura que hasta la década del 70 se atribuía a factores maduracionistas y perceptivistas visuales.

Se ha considerado a la conciencia fonológica, el conocimiento de algunas letras y sus sonidos, la memoria verbal y el lenguaje oral, como aquellas competencias que están relacionadas con el éxito del aprendizaje de la lectura y que, a través de este instrumento, se podrá identificar a aquellos alumnos (as) que presenten deficiencias en estas áreas para que puedan ser intervenidos a tiempo de evitar el fracaso lector.

Uno de los aspectos fundamentales en los hallazgos encontrados es el hecho de haber diseñado una prueba que examina las variables predictoras de la lectura en su conjunto y no de modo aislado, una por una, tal como lo realizan otras pruebas.

De otro lado se encontró que se da la más alta correlación entre conciencia fonémica, en particular las tareas de síntesis fonémica, y la lectura. Esto podría deberse a que el aprendizaje de la lectura en español consiste fundamentalmente en la habilidad de establecer la relación entre el grafema y el fonema, y ello es imposible de lograr sin haber obtenido el nivel más abstracto de la conciencia fonológica, tal como es la conciencia fonémica. En el caso de algunas tareas relacionadas a rimas, se apreció una correlación menor; ello se debería a que dichas tareas no implicarían un nivel de conciencia fonológica muy relacionada a la lectura, debido a que el nivel de reflexión sobre la estructura del lenguaje, en el caso de las rimas, sería muy amplia, no sucediendo así con sílabas y fonemas. Algo similar ocurre con las tareas de memoria: retención y reconocimiento, donde el niño tiene que identificar una figura luego de escuchar la palabra. Esta tampoco tendría mayor grado de asociación con el aprendizaje de la lectura debido a que interviene fundamentalmente la memoria visual y, como sabemos, los nuevos enfoques cognitivos y psicolingüísticos han demostrado que es la memoria verbal, y no la visual, la que se relaciona con el éxito de la lectura.

Por último debemos resaltar que la presente prueba tiene un antecedente directo en la Prueba de predicción lectora: PPL (Bravo, 2000), prueba que evalúa no solo la conciencia fonológica, sino también el lenguaje oral; sin embargo, consideramos que era necesario incluir otras variables de importancia, tales como: identificación de letras y la memoria verbal, otorgándole un mayor rigor técnico y aumentando el número de reactivos. 


\section{CONCLUSIONES}

1. Se ratifica el modelo cognitivo y psicolingüístico de la lectura, que atribuye a la conciencia fonológica, identificación de letras, memoria verbal y lenguaje oral como variables predictores del aprendizaje de la lectura.

2. El Test de habilidades prelectoras (THP) se constituye en un instrumento que cumple con las normas de confiabilidad y validez correspondiente, y que garantiza la evaluación del nivel de dominio en las variables prelectoras.

3. Este instrumento puede ser utilizado no solo con un fin evaluativo, sino, fundamentalmente, con un objetivo preventivo. Proporciona información y orientación sobre las habilidades que deben ser entrenadas antes del aprendizaje lector, previniendo su fracaso y detectando, de manera oportuna, a aquellos niños (as) que presentarán problemas en el aprendizaje de la lectura.

4. Igualmente, el presente Test permitirá perfilar líneas de intervención y recuperación psicopedagógica en las áreas afectadas. De esta manera se podrá remontar las deficiencias cognitivas y psicolingüísticas que subyacen al aprendizaje de la lectura y que se evidencian en los bajos resultados encontrados en las evaluaciones nacionales e internacionales.

\section{NOTA DE RECONOCIMIENTO}

Queremos reconocer y agradecer a los siguientes estudiantes de psicología que colaboraron con eficiencia en nuestro estudio de investigación.

\begin{tabular}{ll}
\hline \multicolumn{1}{c}{ Apellidos y nombres } & \multicolumn{1}{c}{ Lugar de procedencia } \\
\hline 1. Huerta Roca, Edson Martin & Facultad de psicología - UNMSM \\
2. Fernández Cárdenas, Lizet Noemí & Facultad de psicología - UNMSM \\
3. Meléndez Jara, Sandra Patricia & Facultad de psicología - U. Federico Villarreal \\
4. Casariego Luna, Jhasmany Alex Armando & Facultad de psicología - UNMSM \\
5. Cárdenas Medina, Lizet Yamely & Facultad de psicología - U. Federico Villarreal \\
\hline
\end{tabular}

\section{REFERENCIAS BIBLIOGRÁFICAS}

1. Bravo, L. y Orellana, E. (1999). La conciencia fonológica y el aprendizaje de la lectura. En Boletín de Investigación Educacional. Santiago de Chile: Editorial Universidad Católica de Chile.

2. Bravo, L. (2009). Trastornos del aprendizaje: investigaciones psicológicas y psicopedagógicas en diversos países de Sudamérica. En Revista ciencias psicológicas de la Universidad Católica del Uruguay, 3. 
3. Bravo, L. (2005). Lenguaje y dislexia. Enfoque cognitivo del retardo lector. Santiago de Chile: Editorial Universidad Católica de Chile.

4. Bravo, L.; Villalón, M. y Orellana, E. (2000). Nivel de desarrollo fonológico y lectura emergente en niños de escuelas municipales. En Boletín de investigación educacional de la Universidad Católica de Chile, 16, 165-17.

5. Canales, R. (2007). Comprensión lectora y problemas de aprendizaje: un enfoque cognitivo. Lima: Consejo Nacional de Ciencia, Tecnología e Innovación TecnológicaCONCYTEC.

6. Carmines, E. y Zeller, R. (1979). Relibility and validity assessment. California: Sage.

7. Compton, D. (2000). Modeling the growth of decoding skills in first grade children. En Scientific studies of reading, 4, 219-259.

8. Condemarín, M.; Milicic, N. y Alliende, F. (1990). Prueba CLP-Formas paralelas. Santiago de Chile: Universidad Católica de Chile.

9. Cuetos, F. (2008). Psicología de la lectura. España: S.A. Wolters Kluwer.

10. Domínguez, A. (1999). La enseñanza de la lectura. Enfoque psicolingüístico sociocultural. Madrid: Ediciones Pirámide.

11. Ministerio de Educación. (2007). Evaluación censal de estudiantes. Tomado de http:// www.minedu.gob.pe.

12. Gonzalez, R. (2006). Problemas psicolingüísticos en el Perú. Lima: Edic. N. Reátegui.

13. Jiménez, J. E. y Ortiz, G. M. (1998). Conciencia fonológica y aprendizaje de la lectura: teoría, evaluación e intervención. Madrid: Editorial Síntesis.

14. Llece. (2006). Laboratorio latinoamericano de evaluación de la calidad de la educación. Tomado de http://llece.unesco.cl.

15. Ministerio de Educación del Perú. (2001). Una aproximación a la alfabetización lectora de los estudiantes peruanos. Resultados del Perú en la evaluación internacional PISA. En Documento de trabajo, 6.

16. Pinzàs, J. (1997). Metacognición y lectura. Lima: Pontificia Universidad Católica del Perú.

17. Rosales, A. (1984). Desarrollo de mecanismos de lectura comprensiva en niños de poblaciones urbanas. Lima: Asociación Peruana para el Formato de las Ciencias Sociales (FOMCIENCIAS).

18. Tapia, V. (1996). Desarrollo de un programa de lectura en niños deficientes lectores. En Psicología cognitiva y sus aplicaciones a la clínica educacional. Lima: Universidad San Martín de Porres.

19. Treiman, R. (1990). Phonological Awareness and its roles in learning to read and spell. En D. J. Sawye y B. J. Fox (Eds.), Phonological Awareness in reading. The evolution of current perspective, (159-189). New York: Spinger-Verlag. 
20. Velarde, E. (2001). Relación entre la conciencia fonológica y el nivel de decodificación y comprensión lectora en niños de 8 años del tercer grado de primaria de dos niveles socioeconómicos del cercado del Callao (tesis de Maestría). Lima: UNIFÉ.

21. Velarde, E. y Canales, R. (2008). La lectura en el Perú. Drama y esperanza. Lima: Edic. Universidad "Enrique Guzmán y Valle".

22. Velarde, E. (2008). Elaboración y aplicación de un programa metafonológico en niños de 8 a 10 años de tercer y cuarto grado de primaria del Cercado del Callao (tesis doctoral en educación). Lima: UNMSM.

23. Velarde, Canales, Meléndez y Lingán. (2009). Diseño y ejecución de un programa de intervención para el mejoramiento de la lectura en niños de la Provincia del Callao. Ponencia presentada a X Congreso Latinoamericano de Lectura y Escritura, Lima, (paper). 